\title{
FES of the effect of free vibration treatment on fatigue damage recovery for notched copper film
}

\author{
Hai-Meng Zhang, De-Guang Shang*, Shuai Lv \\ College of Mechanical Engineering and Applied Electronics Technology, Beijing University of Technology, Beijing 100124, China
}

\begin{abstract}
In this paper, finite element simulation (FES) of the effect of free vibration treatment on fatigue damage recovery for notched copper film with the thickness of $33 \mathrm{um}$ was investigated. The damage process analyzed by static analysis of original specimen and vibration process analyzed by transient dynamic analysis of damaged specimen were simulated respectively. The transient dynamic analysis was conducted with different frequencies and acceleration amplitudes. It is found that the plastic strain was produced as a result of cyclic loading and reduced after the vibration treatment. The values of plastic strain are different after treatment with different vibration frequencies and acceleration amplitudes. Furthermore, the plastic strain in notch reduces more than that of other areas of the specimen. According to the analysis, the vibration treatment can reduce the plastic strain of notched specimen significantly by which the microproperties are improved so that fatigue damage can be recovered.
\end{abstract}

\section{Introduction}

The VSR (vibratory stress relief) process is a green and emerging technology that refers to the relaxation of residual stress via cyclic loading treatments. VSR methods have been successfully used to relieve or redistribute stress and therefore guard against service failures, such as stress corrosion cracking. Heat treatment is a popular stress relief method in the metal forming industry. While this treatment yields stress-free materials, it has numerous disadvantages, such as along process time, significant energy consumption, oxide scale formation on the surface, and substantial effects on the mechanical properties. VSR does not suffer from these disadvantages. Although the stress relief performance of the VSR process remains inferior to that of heat treatment, this method is widely used in various industrial fields as a result of its time-saving, small equipment volume requirements, low cost, minimal energy consumption [1-5].

The relationship between VSR and fatigue damage recovery have been studied by many researchers. A. S. M. Y. Munsi et al. [6] investigated the effect of VSR treatment on fatigue life improvement for weld components. The results indicated that the fatigue life of welds increased 17\%-30\% after VSR. D.L. Rao et al. [7] found that the residual stress measurement results show that the average principal stresses decrease by about $56 \%$ for the drum of hoist machine and decrease by about $31 \%$ for the stainless steel 304L plate after VSR. Y.P. Lu et al. [8] compared the mechanisms of VSR and TSR(thermal stress relief). They indicated that both methods are similar in reducing macroscopic residual through plasticity deformation to reduce residual elasticity strain. Y.D. Zhang et al. [9-11] investigated the effect of VSR. That low amplitude VSR saves more energy than the high amplitude VSR with similar stress relieving effect. They believed that low amplitude VSR has positive influences on the fatigue whereas high amplitude VSR has negative influences. M. Jafari et al. [12] proposed a mathematical model based on plasticity theorem with linear kinematic hardening to explain the mechanism of reduction of residual stresses of materials experiencing cyclic loading. The numerical results from the mathematical model and the analytical results with existing experimental data for rectangular cross-section beams showed a similar trend. However, D. Djuric et al. [13] found that no clear tendencies could be seen. Nevertheless, fatigue damage due to the VSR treatment could be found. The changes in residual stresses did not cause macroscopic deformations of the specimens.

Since one end of the specimen was clamped to a vibration stage with fixture, and the other end is free to move in the method mentioned above, it may induce additional damage which similar with vibration fatigue during treatment.

Finite element method has been widely used to investigate mechanical properties of materials and structures. It can be used to simulate various test schemes and optimal design to reduce test time and expense [14-16].

The aim of this paper is to investigate the effect of unconstraint vibration treatment on fatigue damage recovery by means of finite element simulation which is a preliminary study for improving fatigue life. Both two ends of the specimen are free to move during vibration treatment and the direction of vibration is perpendicular

* Corresponding author: author@e-mail.org 
to the longitudinal direction. The effects of vibration parameters including different vibration frequency and vibration acceleration amplitude on plastic strain reduction are also analysed.

\section{Simulated cases}

A notched copper film model is simulated in this investigation. The shape and dimensions of the copper film specimen are shown in Fig.1.

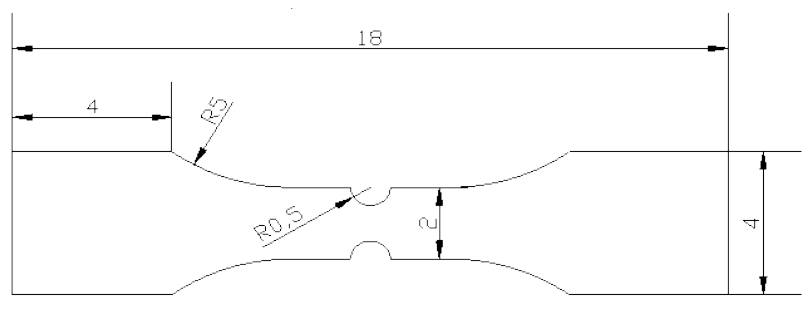

Fig. 1. Shape and dimensions of copper film specimen.

The element type selected for the specimen is shell 181. Meanwhile, a combination element named combin 14 is also selected to apply constrains since the analysis process cannot conduct without any constrains on the model in transient dynamic analysis. The parameters of the real constants of the combination element are shown in table 1.

Table 1. Parameters of the combination element

\begin{tabular}{|c|c|}
\hline $\begin{array}{c}\text { Spring } \\
\text { constant }\end{array}$ & 10 \\
\hline $\begin{array}{c}\text { Damping } \\
\text { coefficient }\end{array}$ & 0.02 \\
\hline
\end{tabular}

Combined nonlinear isotropic-kinematic hardening is used to describe the elastic-plastic material behaviour. The material parameters of the specimen are listed in Table 2.

Table 2. Material parameters of the specimen

\begin{tabular}{|c|c|}
\hline $\begin{array}{c}\text { Young's } \\
\text { modulus(MPa) }\end{array}$ & 42000 \\
\hline Poisson's ratio & 0.3 \\
\hline $\begin{array}{c}\text { yield } \\
\text { strength(MPa) }\end{array}$ & 252 \\
\hline $\begin{array}{c}\text { tensile } \\
\text { strength(MPa) }\end{array}$ & 418 \\
\hline $\begin{array}{c}\text { elongation(\%) } \\
\text { elo }\end{array}$ & 6 \\
\hline
\end{tabular}

\subsection{Meshing}

The element edge length of the model is $0.0001 \mathrm{~m}$ and division arc of the notch of the model is $3^{\circ}$. The model is convergent by choosing these parameters. Meshing of the model after free mesh is shown in Fig.2.

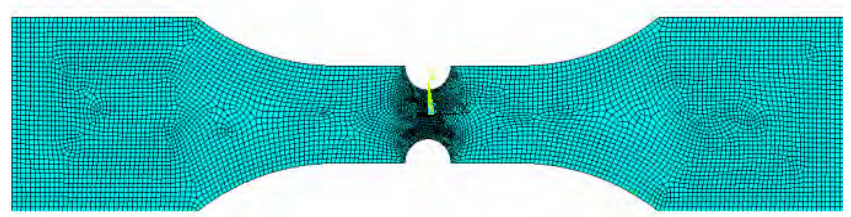

Fig. 2. Meshing of copper film specimen.

\subsection{Loading}

The damage process analyzed by static analysis of original specimen and vibration process analyzed by transient dynamic analysis of damaged specimen are simulated respectively. A transverse non-load-carrying attachment subjected to axial loading is modeled using elastic-plastic finite elements.The force appiled to the axial direction in fatigue process is $9 \mathrm{~N}$. Two cycles including four load steps are loaded and each load step including one substep. The load-time history is shown in Fig.3. The axial direction is the $\mathrm{X}$ direction and width direction is the $\mathrm{Y}$ direction during analysis.

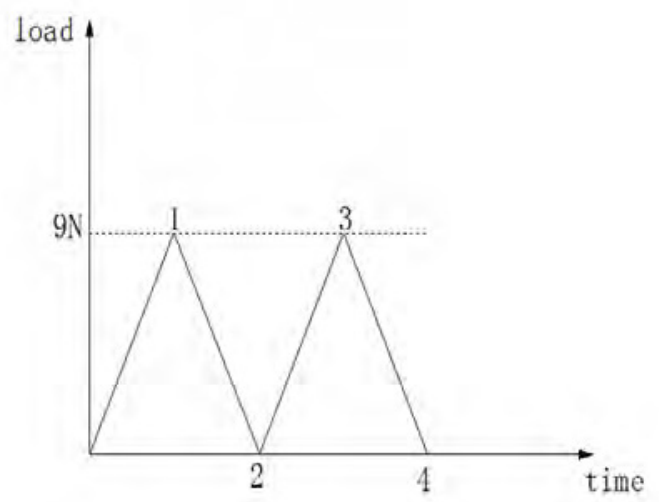

Fig. 3. Load-time history of fatigue process.

A new node perpendicular to the specimen center is created, then it is a combined with the node in the center of the specimen as a combination element. Displacement constraints were applied on the new node. A prestress which produced in fatigue damage process is guided into the model before transient dynamic analysis is conducted. The specimen is excited under a sinusoidal vibration waveform which is perpendicular to the XY plane and action time is one cycle. A vibration data file which consists of vibration time and vibration acceleration is guided into the model as the loading during transient dynamic analysis. The load-time history of vibration process is shown in Fig.4. Different time represents different vibration frequencies in the data file. 


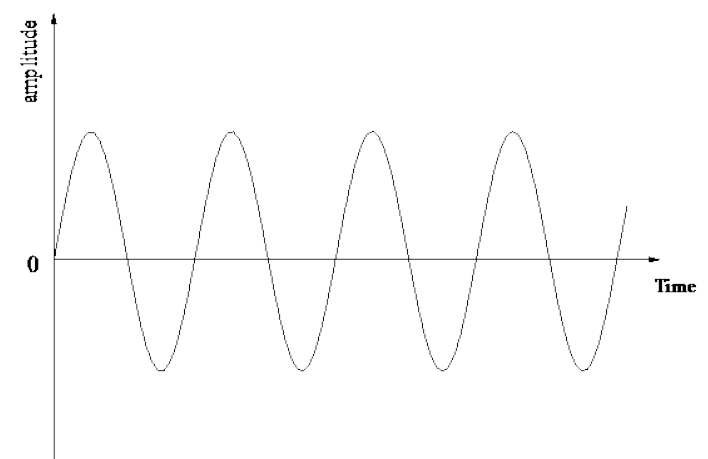

Fig. 4. Load-time history of vibration process.

\section{Results and discussion}

The X directional plastic strain distribution of the notch area after fatigue loading is shown in Fig 5.

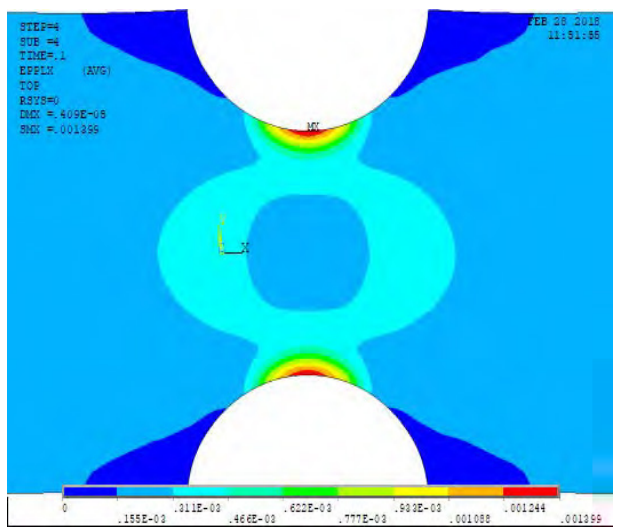

Fig. 5. X direction plastic strain distribution of the notch area after fatigue loading.

The results show that the maximum plastic strain is located at the root of specimen notch which was the stress concentration district and the value is $1.399 \times 10^{-2}$. In general, it is superior to initiate fatigue crack in this district.

\subsection{Effect of vibration frequency}

Vibration process is simulated under different vibration frequencies including $40 \mathrm{~Hz}, 60 \mathrm{~Hz}, 80 \mathrm{~Hz}, 100 \mathrm{~Hz}, 150 \mathrm{~Hz}$ respectively. All of the vibration acceleration amplitude applied in these simulations is $4 \mathrm{~g}$. The $\mathrm{X}$ directional plastic strain distribution of the notch is shown in Fig 6 . Different colours represent different strain values, and the value of the strain in red region is maximum.

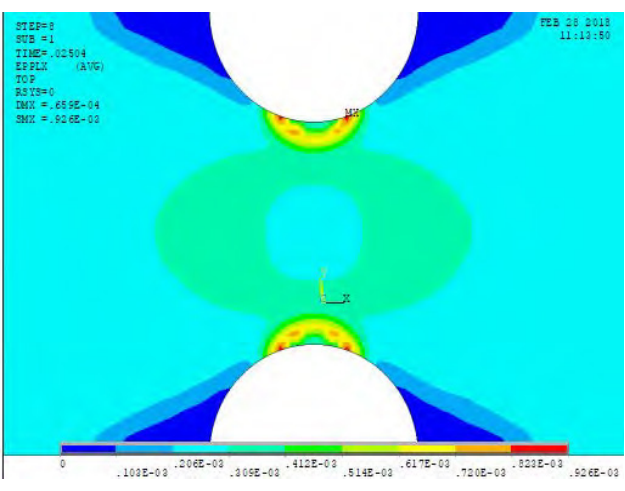

(a)

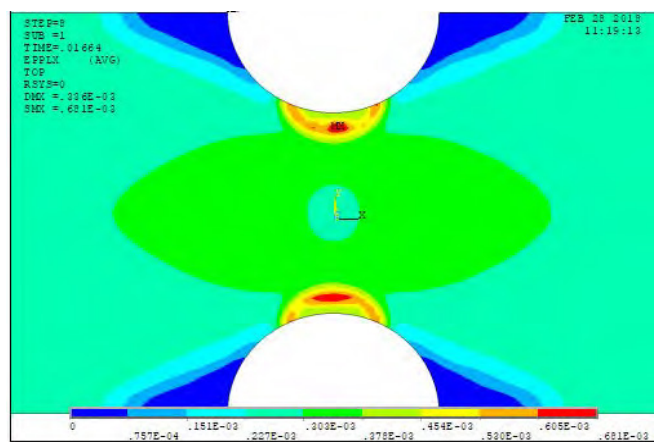

(b)

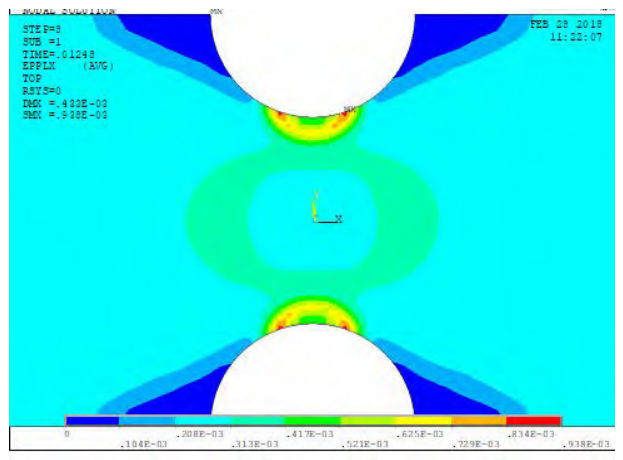

(c)

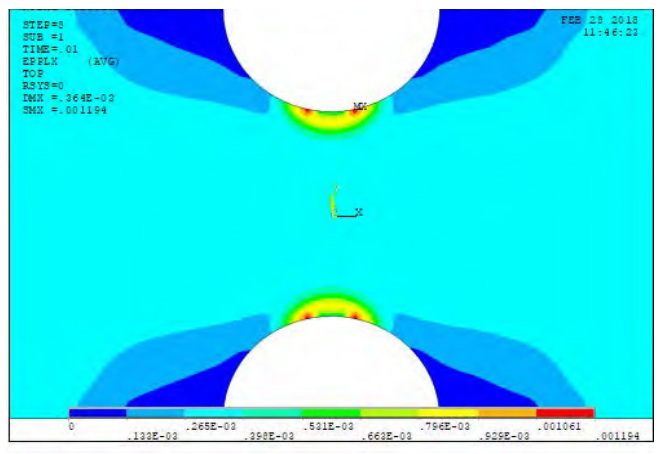

(d) 


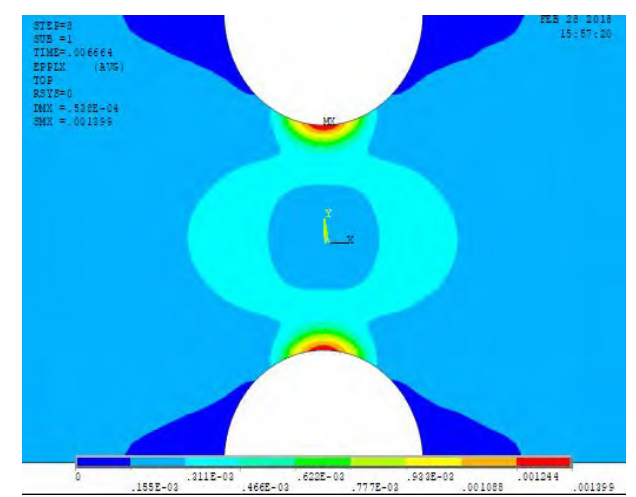

(e)

Fig.6. $\mathrm{X}$ directional plastic strain distribution of the notch area (a) $40 \mathrm{~Hz}$, (b) $60 \mathrm{~Hz}$, (c) $80 \mathrm{~Hz}$, (b) $100 \mathrm{~Hz}$, (e) $150 \mathrm{~Hz}$.

The maximum value of the $\mathrm{X}$ directional plastic strain under different vibration frequencies in the notch is shown in Fig 7.

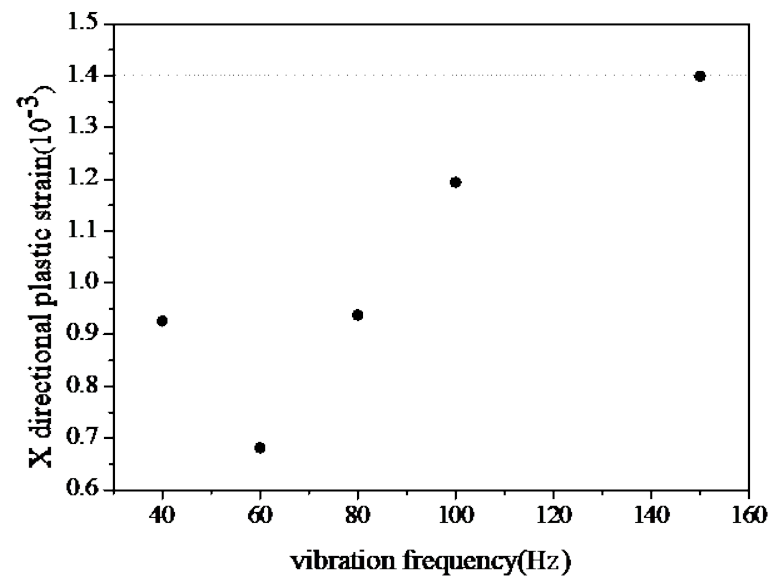

Fig.7. Maximum value of the $X$ directional plastic strain under different vibration frequencies.

As can be seen in the figure 7 , all of the $\mathrm{X}$ directional plastic strain are decreased after vibration treatment expect $150 \mathrm{~Hz}$ compared to that without vibration treatment and the value of the plastic strain is $0.926 \times 10^{-3}$, $0.681 \times 10^{-3}, \quad 0.938 \times 10^{-3}, \quad 1.194 \times 10^{-2}, \quad 1.399 \times 10^{-2}$, respectively. The results show that $\mathrm{X}$ directional plastic strain decreases firstly and then increases with the increase of vibration frequency and the value is minimum when the vibration frequency is $60 \mathrm{~Hz}$. The plastic strain decreases only if with proper vibration frequency, the plastic strain cannot decrease even increase with other vibration frequencies. Frequency is related to the mode of vibration. When the vibration frequency is low, vibration is irregular according to the results of simulation which may affect the effect of vibration treatment on fatigue damage recovery, so that the reduction of plastic strain is little. When the vibration frequency is too high, the mode of vibration cannot produce effective stress to recover damage defect, so that the plastic strain does not decrease.

\subsection{Effect of vibration acceleration amplitude}

Vibration process under different vibration acceleration amplitudes is simulated including $3 \mathrm{~g}, 4 \mathrm{~g}, 5 \mathrm{~g}, 6 \mathrm{~g}, 7 \mathrm{~g}$, respectively. All of the vibration frequencies applied is $60 \mathrm{~Hz}$. The $\mathrm{X}$ directional plastic strain distribution of the notch is shown in Fig 8. Different colours represent different strain values, and the strain of red region is maximum.

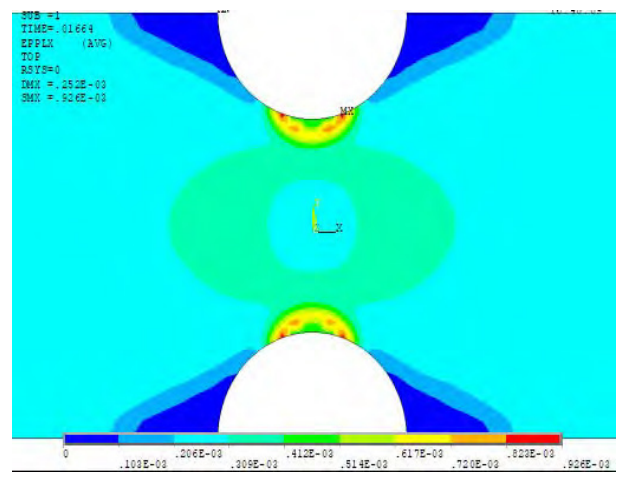

(a)

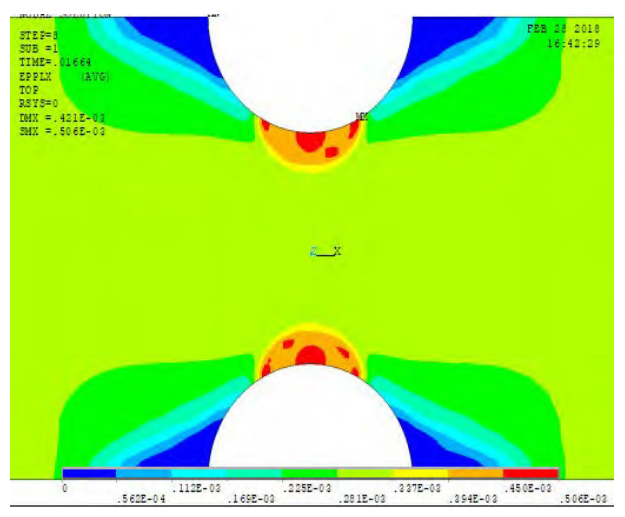

(b)

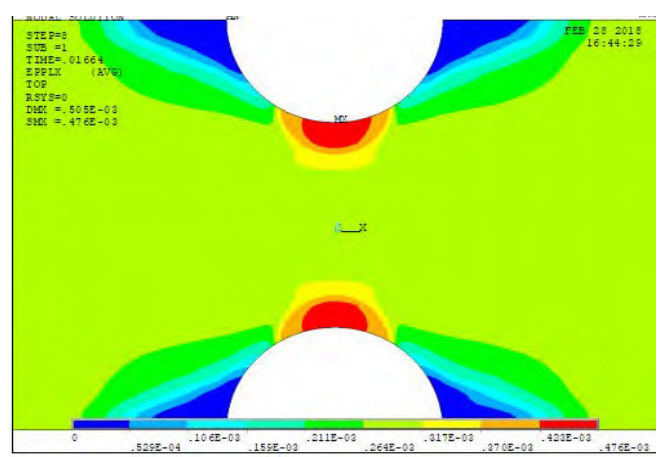

(c) 


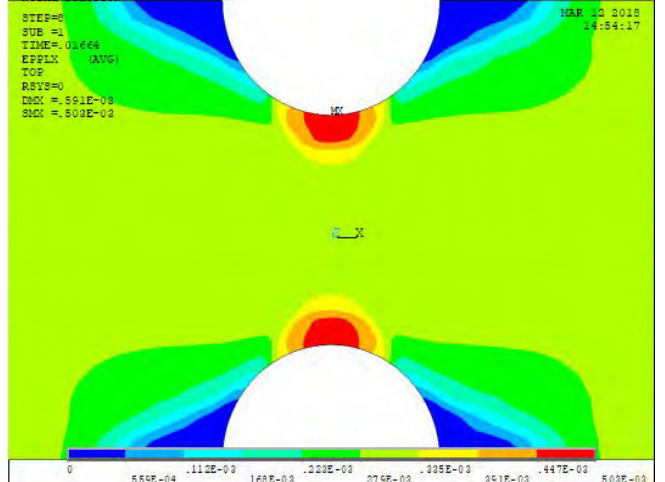

Fig. 8. X directional plastic strain distribution of the notch area (a) $3 \mathrm{~g}$, (b) $5 \mathrm{~g}$, (c) $6 \mathrm{~g}$, (d) $7 \mathrm{~g}$.

It can be found that the maximum plastic strain point is located at the verge of the specimen notch but not in the stress concentration district when the vibration acceleration amplitude is $3 \mathrm{~g}$. The maximum plastic strain point is located inside the verge of the specimen notch and away from the stress concentration district when the vibration acceleration amplitude is $4 \mathrm{~g}$ as listed in figure 6 . However, the maximum plastic strain point is located at the verge of the specimen notch and also in stress concentration district when the vibration acceleration amplitudes are from $5 \mathrm{~g}$ to $7 \mathrm{~g}$.

Values of the $\mathrm{X}$ directional plastic strain of the notch root under different vibration acceleration amplitudes are shown in Fig 9.

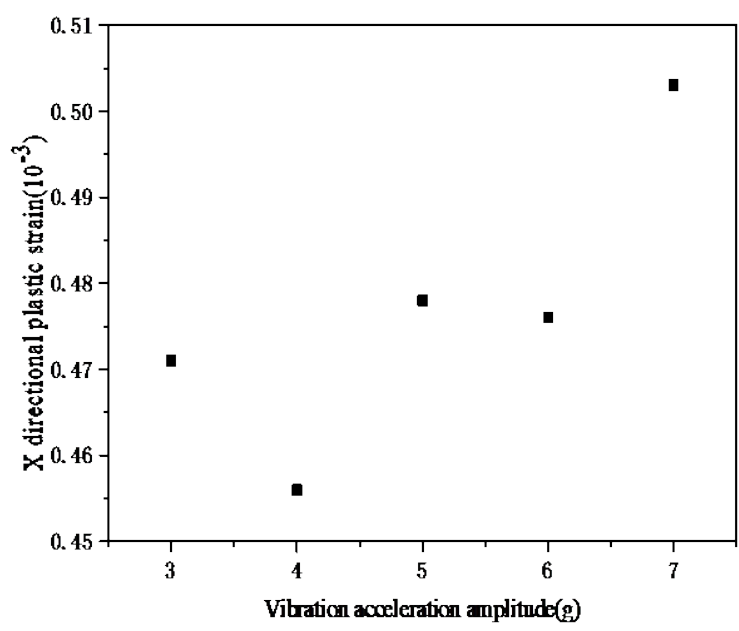

Fig. 9. Values of the $X$ directional plastic strain of the notch root under different acceleration amplitudes.

As can be seen in the figure 9, all of the $\mathrm{X}$ directional plastic strain of the notch root are decreased after vibration treatment compared to that without vibration treatment and the value of the $\mathrm{X}$ directional plastic strain is $0.471 \times 10^{-3}, 0.456 \times 10^{-3}, 0.478 \times 10^{-3}, 0.476 \times 10^{-3}$, $0.503 \times 10^{-3}$, respectively. The results show that $X$ directional plastic strain decreases firstly and then increases with the increase of vibration acceleration amplitude and the value is minimum when the vibration frequency is $4 \mathrm{~g}$. Vibration acceleration amplitude is related to the height of specimen vibration and the height is related to the exciting force during vibration. The plastic strain decreases only if with proper vibration acceleration amplitude, the plastic strain cannot decrease even increase with other vibration acceleration amplitudes. Too little exciting force is not enough for the driving force to eliminate the dislocation and too much force will produce additional damage.

Compared to conventional VSR which one end of the specimen is champed to a vibration stage with fixture and the other end is free to move, both ends are free in the approach proposed in the present study and could avoid additional damage which is induced during conventional VSR.

Simulation results indicate that the proposed method is effective on fatigue damage recovery. Vibration treatment with proper parameters can produce exciting force which acts as a driving force that return the internal structure to a stable state. It is known that the energy of plastic strain is stored in dislocation structures during fatigue. Plastic strain decrease even disappear means dislocation decrease and microstructure improvement so that fatigue crack initiation is delayed and fatigue life is improved.

\section{Conclusions}

In this work, finite element method is utilized to derive the change of the notched copper film before and after unconstraint vibration treatment with different frequencies and different acceleration amplitudes. The conclusions are as follows:

1. The plastic strain of the specimen decreases effectively and the reduction is different after vibration treatment with different frequencies and different acceleration amplitudes. The optimal parameters are $60 \mathrm{~Hz}$ and $4 \mathrm{~g}$ respectively.

2. Vibration frequency determines the value of the axial direction plastic strain and vibration acceleration amplitude determines the site of maximum value of the axial direction plastic strain.

3. With the decrease of the plastic strain, the microstructure of the specimen is improved and the specimens are in a stable state which is good for delaying fatigue crack initiation and improving fatigue life.

The authors would like to acknowledge the support of the financial support of the National Natural Science Foundation of China(No.51535001,11572008)

\section{References}

1. J.S. Wang, C.C. Hsieh, C.M. Lin, E.C. Chen, C.W. Kuo, W.T. Wu. Materials Science\&Engineering A. 605 (2014)

2. J.S. Wang, C.C. Hsieh, C.M. Lin, E.C. Chen, C.W. Kuo, W.T. Wu. metallurgical and Materials Transactions 44A (2013)

3. J.S. Wang, C.C. Hsieh, H.H. Lai, C.W. Kuo, Y. Wu P. Ti, W.T. Wu. Materials Characterization 99 (2015) 
4. D. Rao, D. Wang, L. Chen, C. Ni. International Journal of Fatigue 29 (2007)

5. C.M. Sonsino, F. Muller, J De Back, A. M. Gresn. Fatigue Fract Eng M 19, 6 (1996)

6. A. S. M. Y. Munsi, A. J. Waddell, C. A. Walker. Strain 37, 4 (2001)

7. D.L. Rao, Z.Q. Zhu, Chen L.G, C.Z. Ni. Materials Science Forum 490-491 (2005)

8. Y.P. Lu, Z.Y. Ma, Q.S. Jia, R.R. Gu. Mechanical Science and Technology 20 (2001)

9. J. Song, Y.D. Zhang. Advances in Mechanical Engineering 8, 6 (2016)

10. H.J. Gao, Y.D. Zhang, Q. Wu, J. Song. Metals 7 (2017)

11. H.J. Gao, Y.D. Zhang, Q. Wu, J. Song, K. Wen. International Journal of Fatigue 108 (2018)

12. M. Jafari Vardanjani, M. Ghayour, R. Mokhtari Homami. Experimental Techniques 40 (2016)

13. D. Djuric, R. Vallant, K. Kerschbaumer, N. Enzinger. Welding in the World 55 (2011)

14. J. Lee, J. Kim, S. Kang, M. Hirohata, K. Chang. International Journal of Steel Structures 172 (2017)

15. S.Q. Li, H.Y. Fang, X.S. Liu, W. Wang, Q. Wang, W. Cui. Journal of vibroengineering 183 (2016)

16. M.M. El-Sayed, A.Y. Shash, M. Abd-Rabou. Journal of Materials Processing Tech 252 (2018) 